\title{
Modelagem e previsão de vazões médias mensais do Rio Paranaíba utilizando o modelo SARIMA
}

Devido ao crescimento desordenado das cidades, a expansão da industrialização e do agronegócio, o Brasil já enfrenta problemas de escassez hídrica. A gestão dos recursos hídricos é de suma importância para minimizar os transtornos causados por esse tipo de problema. Neste sentido, a previsão de vazões tem importância significativa para o gerenciamento dos recursos hídricos, com destaque para os modelos estocásticos de séries temporais, que vêm sendo utilizados para tal. No presente trabalho utilizaram-se modelos da classe SARIMA, para a previsão de uma série temporal de vazões médias mensais de uma sub-bacia do Rio Paranaíba na região do Alto Paranaíba, MG. A metodologia adotada foi a de Box e Jenkins e a identificação da ordem dos modelos foi feita através da análise gráfica das funções de autocorrelação (FAC) e autocorrelação parcial (FACP). Para a seleção do modelo foram utilizados critérios de seleção AIC e BIC de tal modo que, dentre os modelos selecionados, o modelo SARIMA $(2,1,2)(1,0,0) 12$ foi o que melhor se ajustou aos dados em estudo. 0 referido modelo obteve um NS satisfatório de 0,85 para a calibração realizada de 2003 a junho de 2014. Já a previsão para 6 meses de 2014, o NS fora de -3,34, demonstrando uma não adequação do modelo aos dados observados de vazão. Tal fato se justifica pela crise hídrica observada na região no ano de 2014 . 0 estudo demonstra que há situações que os modelos estatísticos não conseguem prever períodos de estiagem dado ao fato destes não fazerem parte do domínio de dados previamente estabelecido.

Palavras-chave: Recursos hídricos; Modelagem hidrológica; ARIMA; Séries temporais.

\section{Modeling and forecasting mean monthly streamflows of Paranaiba River using SARIMA model}

\begin{abstract}
Due to the disorderly growth of cities, industrialization and agricultural expansion, Brazil is already beginning to face problems of water scarcity. The management of water resources is of paramount importance to minimize the inconvenience caused by this type of problem. In this sense, the forecast streamflows has a significant importance in the management of water resources and are essential for a good planning and control of water uses. For that, stochastic models of time series can be used to determine the streamflows forecasts. In this work, models of the SARIMA class were used, as they incorporate seasonal characteristics in the model. The objective was to model and carry out forecasting studies of a time series of mean monthly streamflows in a Paranaiba River basin in the Alto Paranaíba region, MG, with data from January 2003 to December 2014. The methodology adopted was that of Box and Jenkins and identification of the order of the models was done through the graphic analysis of the autocorrelation (ACF) and partial autocorrelation functions (PACF). For the model selection, AIC and BIC selection criteria were used, in such a way that, among the selected models, the SARIMA model $(2,1,2)(1,0,0) 12$ was the one that best fit the data in study. This model obtained a satisfactory NS of 0.85 for the calibration. As for the validation of the forecast, for a 6 -month ahead, the NS outside -3.34 , showing that the model did not fit the data presented. This fact points to the water crisis observed in the region in 2014. The study shows that there are situations that statistical models may not be able to predict periods of drought, because they are not part of the previously established data domain.
\end{abstract}

Keywords: Water resources; Hydrological modelling; ARIMA; Time series.

Topic: Engenharia de Recursos Hídricos

Reviewed anonymously in the process of blind peer.
Received: 05/03/2021

Approved: $23 / 03 / 2021$
Lorena Taís Caixeta

Universidade Federal de Viçosa, Brasil

http://lattes.cnpq.br/9651738861498339

http://orcid.org/0000-0002-1391-0877

lorenataiscaixeta@gmail.com

Frederico Carlos Martins de Menezes Filho (iD)

Universidade Federal de Viçosa, Brasil

http://lattes.cnpq.br/6934146250991610

http://orcid.org/0000-0003-4874-0254

frederico.menezes@ufv.br

Vitor Luís Amorim Fonseca

Universidade Federal de Viçosa, Brasi

http://lattes.cnpq.br/9404070525173256

vitor.amorimciivil@gmail.com

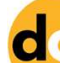

DOI: 10.6008/CBPC2179-6858.2021.003.0022
Referencing this:

CAIXETA, L. T.; MENEZES FILHO, F. C. M.; FONSECA, V. L. A..

Modelagem e previsão de vazões médias mensais do Rio Paranaíba utilizando o modelo SARIMA. Revista Ibero Americana de Ciências Ambientais, v.12, n.3, p.255-267, 2021. DOI:

http://doi.org/10.6008/CBPC2179-6858.2021.003.0022 


\section{INTRODUÇÃO}

Em um relatório publicado em 2019, a Organização das Nações Unidas para a Educação, a Ciência e a Cultura (UNESCO, 2019), adverte que o uso de água em nível global aumentou seis vezes nos últimos 100 anos e permanece crescendo a uma taxa de $1 \%$ ao ano. $O$ referido documento relata que a combinação de um abastecimento ineficiente com as mudanças climáticas tem agravado problemas de escassez hídrica no mundo.

No Brasil, a disponibilidade hídrica gira em torno de $12 \%$ do total da água doce do planeta, no entanto, acompanhando a tendência mundial, o estresse hídrico vem aumentando com o passar dos anos, em decorrência do aumento da população e das atividades econômicas intensivas em uso de água. De acordo com a Conjuntura dos Recursos Hídricos no Brasil no ano de 2019, desenvolvido pela Agência Nacional de Águas (ANA, 2019), a demanda por uso de água no Brasil é crescente e tem um aumento estimado de, aproximadamente, $80 \%$ no total de água retirada nas últimas duas décadas.

Atualmente, o principal uso de água no país, em termos de quantidade utilizada, é a irrigação. A Agência Nacional das Águas (ANA, 2019) aborda que, no ano de 2019, somente a irrigação agrícola consumiu $728 \mathrm{~m}^{3} / \mathrm{s}$, dos $1101 \mathrm{~m}^{3} / \mathrm{s}$ do total de água consumida no Brasil, representando $66,1 \%$. O citado documento apresenta que tal uso supera a soma do abastecimento público, do consumo das indústrias e da mineração. Neste contexto, o estado de Minas Gerais concentra 30,6\% da área equipada por pivôs, sendo o principal estado no ramo (ANA, 2019).

A bacia do rio Paranaíba, localizada na região do Alto Paranaíba em Minas Gerais, apresenta demanda de água para atividades de agricultura irrigada, dessedentação animal, abastecimento humano, mineração e indústria (Comitê da Bacia Hidrográfica dos Afluentes Mineiros do Alto Paranaíba - CBH AMAP, 2013). A Figura 1 demonstra a composição relativa das demandas setoriais por consumo de água. Assim, pela diversidade de atividades de uso extensivo da água, em conjunto com a estiagem, geraram uma escassez hídrica no ano de 2014 na região.

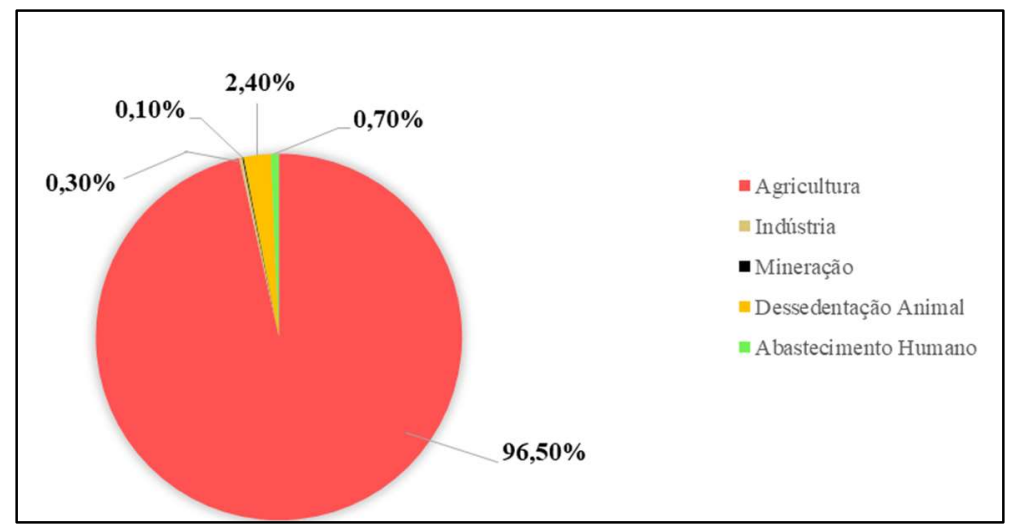

Figura 1: Composição relativa das demandas setoriais por água. Fonte: Adaptado CBH AMAP (2013).

De acordo com Pinto et al. (2015), para entender a dinâmica do ciclo da água nas bacias hidrográficas, é necessário estudar as vazões e representar a resposta da bacia frente às alterações climáticas e antrópicas. O mencionado autor aponta que, a previsão de vazões tem uma significativa importância no gerenciamento 
dos recursos hídricos. Essas previsões podem ser realizadas tanto em curto prazo, geralmente utilizando modelos determinísticos, como a longo prazo, utilizando modelos estocásticos (COLLISCHONN et al., 2005). Segundo Tucci (1998), os modelos determinísticos podem ter solução em equações empíricas e os modelos estocásticos fazem a consideração da probabilidade.

Neste enfoque, as previsões de curto prazo, como abordam Barbosa et al. (2001), são direcionadas para o objetivo de otimizar a operação dos recursos hídricos como controle de cheias, geração de energia elétrica e navegação, ou seja, os usos múltiplos. Já as previsões de longo prazo, conforme Collischonn et al. (2005), têm o foco em antecipar a disponibilidade hídrica, essencial para um planejamento e controle de qualidade dos recursos hídricos.

Para se determinar as previsões de vazão é necessária uma série temporal. Morettin et al. (2006), definem série temporal como um conjunto de observações feitas, sequencialmente, ao longo do tempo. Estas são descritas por uma função matemática (série determinística) ou por distribuições de probabilidade (processo estocástico). A adequabilidade do modelo estatístico é verificada em função da capacidade de ajustar as principais características de uma série temporal, denominadas como tendência e sazonalidade, isto é, um padrão de repetição nos dados.

Dentre os modelos estocásticos, os modelos de séries temporais da classe Autorregressivo Integrado e de Médias Móveis, denominados ARIMA, possuem grande utilização. Por exemplo, para previsões de temperaturas médias, umidade relativa do ar, ou até número mensal de casos de dengue (CHECHI et al., 2012; WERNER et al., 2017; LIZZI, 2012).

Segundo Bayer et al. (2012) estes modelos são, historicamente, utilizados de maneira satisfatória de forma a caracterizar o comportamento de variáveis hidrológicas. Porém, em estudos de variáveis ambientais é comum encontrar sazonalidades nas séries. Nesses casos, verifica-se a necessidade de utilizar modelos que incorporem essa característica sazonal e, assim, há a ampliação dos modelos ARIMA para os modelos SARIMA.

Martínez et al. (2020) utilizaram modelos do tipo ARIMA para modelar vazões médias mensais do rio Sinú, que está situado na região noroeste da Colômbia. Os resultados indicaram que o modelo sazonal modelado atendeu a todos os testes e se mostrou com o melhor desempenho. Em outro estudo, apresentando por Khairuddin et al. (2019), foram utilizados os modelos do tipo ARIMA e Redes Neurais Artificias (RNA) para modelar vazões médias mensais do rio Muda, que está situado na região norte da península da Malásia. Os resultados encontrados pelos autores demonstram que os resultados do método de RNA foram mais eficientes para reconhecer os padrões de séries temporais e suas relações de não linearidade.

Estudos brasileiros para previsões de vazões revelaram a eficiência do método SARIMA, ao se ajustar às séries observadas. Bayer et al. (2012) utilizaram modelos do tipo SARIMA para modelar as vazões médias do rio Potiribu, localizado na região noroeste do estado de Rio Grande do Sul. O modelo escolhido foi capaz de incorporar características como sazonalidade, correlação serial e se ajustou bem aos valores previstos. Santos et al. (2016) utilizaram o método ARIMA para previsão da vazão afluente mensal do Rio Tocantins, 
onde fica localizada a Usina Hidrelétrica de Tucuruí. Foi concluído, no estudo, que o método SARIMA foi capaz de captar a variabilidade da série de vazão e se mostrou eficiente na previsão. Porém, os autores apontam, como ponto de melhoria do método, o aprimoramento da qualidade das estimativas na área de abrangência de hidrelétricas em períodos de seca e cheia.

Por outro lado, em alguns estudos, a modelagem de vazões utilizando modelos ARIMA apresentou resultados inconsistentes, demostrando pontos de falhas do método. Bleidorn et al. (2019) utilizaram o método para a modelagem de vazões médias mensais do Rio Jucu (ES). O método foi escolhido para previsões com horizonte para todo o ano de 2015, incorporando a sazonalidade da série. Entretanto, os resultados previstos divergiram com os observados, pois, como relatado pelos autores, durante o período observado, a bacia do Rio Jucu passou por uma crise hídrica. Assim, o modelo faz suas previsões a partir de eventos anteriores, não levando em consideração crises que podem ocorrer durante o período.

Diante do exposto, o objetivo deste trabalho foi analisar, modelar e fazer previsões da série temporal de vazões médias mensais de uma sub-bacia do rio Paranaíba, MG. Para a análise foram considerados modelos sazonais da classe ARIMA, conhecidos como modelos SARIMA.

\section{MATERIAIS E MÉTODOS}

A metodologia proposta neste trabalho divide-se em seis etapas, que são ilustradas no fluxograma da Figura 2.

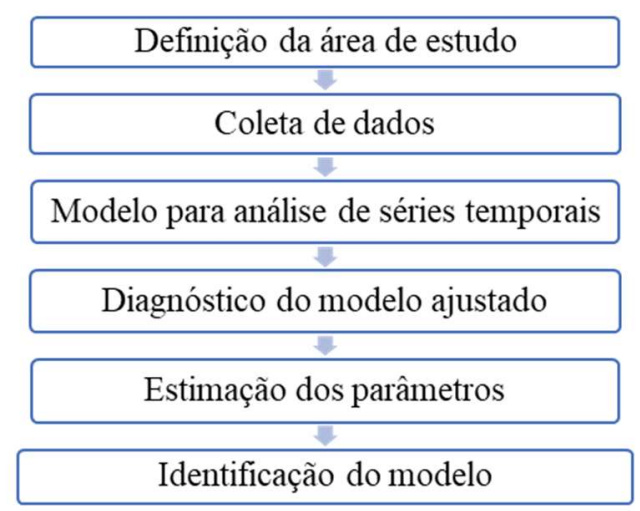

Figura 2: Fluxograma da metodologia.

\section{Área de estudo}

A bacia hidrográfica do rio Paranaíba está posicionada na região central do Brasil e percorre os estados de Minas Gerais, Goiás, Mato Grosso do Sul e Distrito Federal. Sua nascente está situada na Serra da Mata da Corda, no município de Rio Paranaíba, MG, possuindo altitude de, aproximadamente, $1.100 \mathrm{~m}$ e segue até a confluência com o rio Grande, exutório da bacia, para formar o rio Paraná. A bacia do rio Paranaíba possui área de drenagem de $222,6 \mathrm{mil} \mathrm{km}{ }^{2}$, sendo que parte desta área está localizada na região do Alto Paranaíba, no estado de Minas Gerais (Instituto Mineiro de Gestão das Águas - IGAM, 2015).

O estudo foi realizado em uma sub-bacia do Rio Paranaíba, contida na Bacia Hidrográfica dos Afluentes Mineiros do Alto Paranaíba - PN1. A área de estudo está localizada na mesorregião do Alto Paranaíba, em Minas Gerais, com seção de controle na estação fluviométrica Ponte Vicente Goulart, 
totalizando uma área de aproximadamente $7640 \mathrm{~km}^{2}$ (Figura 3).

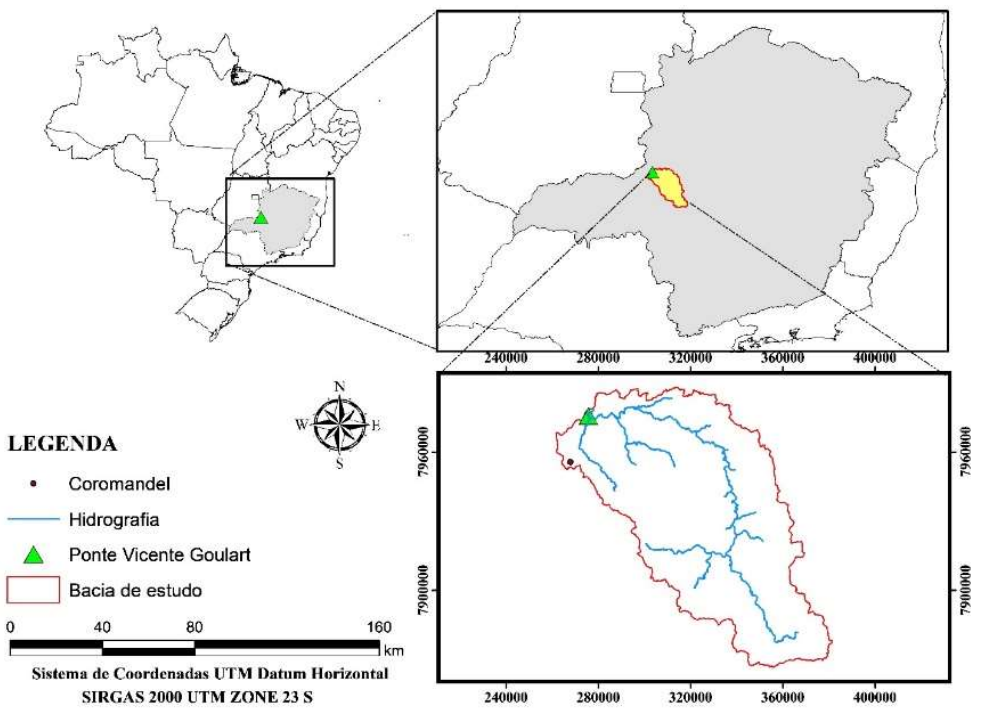

Figura 3: Localização da estação Ponte Vicente Goulart e área de drenagem da bacia.

\section{Coleta de dados}

A série histórica de vazões médias mensais da estação fluviométrica Ponte Vicente Goulart foi obtida junto ao Sistema de Informações Hidrológicas, portal HIDROWEB ${ }^{1}$, mantido pela ANA. A referida série histórica de vazões compreende dados do período de janeiro de 2003 até dezembro de 2014, resultando, assim, em uma série histórica de 12 anos. Para os procedimentos do modelo os dados foram divididos em duas partes: treino e teste. A etapa de treino compreendeu o período de 2003 a junho de 2014 e a etapa de teste, ou seja, fase de validação do modelo, abrangeu o período de julho a dezembro de 2014.

\section{Modelo para Análise de Séries Temporais}

Os cálculos e ajustes necessários para a elaboração deste trabalho foram executados nos Softwares R Studio e Microsoft Excel. Para a modelagem no Software R Studio, utilizaram-se pacotes com funções específicas para análise de séries temporais, como o pacote forecast e stats,

Após a coleta de dados de vazões da estação fluviométrica Ponte Vicente Goulart, realizou-se uma análise descritiva da série temporal. Segundo Chechi et al. (2012), a análise preliminar é importante para a caracterização da série e identificação de sazonalidade, de tendências e valores atípicos. A seguir, foram utilizados os modelos da classe ARIMA, seguindo a metodologia Box \& Jenkins (Box et al., 2008), consoante as etapas apresentadas na Figura 5.

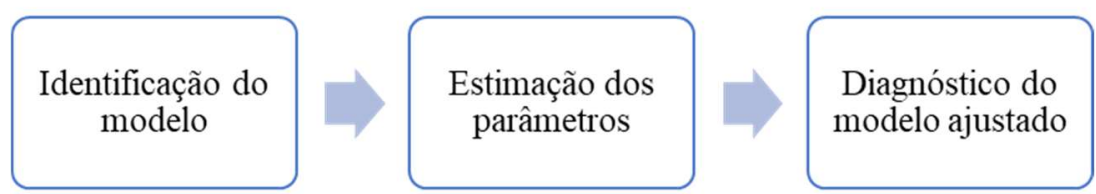

Figura 5: Fluxograma metodologia Box \& Jenkins. Fonte: Adaptado de Box et al. (2008).

\footnotetext{
${ }^{1}$ www.snirh.gov.br/hidroweb/
} 
Para identificação do modelo, de acordo com Morettin et al. (2006), é necessário analisar a estacionariedade do processo. Os processos estacionários são uma importante classe dos processos estocásticos. Assim, se $X_{t}$, uma variável aleatória, seguir um processo autorregressivo e de médias móveis de ordens $p$ e $q, \operatorname{ARMA}(p, q)$, logo a equação 1 será utilizada.

$$
\phi(B) X_{t}=\theta(B) \varepsilon_{t}
$$

Onde $\varepsilon_{t}$ é descrito como ruído branco, sendo um processo puramente aleatório com média zero e variância $\left(\sigma^{2}\right)$. Os parâmetros $\phi$ e $\vartheta$ são, respectivamente, autorregressivos e de médias móveis e são apresentados nas equações 2 e 3.

$$
\begin{aligned}
& \phi(B)=1-\phi_{1} B-\phi_{2} B^{2}-\cdots-\phi_{p} B^{p} \\
& \vartheta(B)=1-\theta_{1} B-\theta_{2} B^{2}-\cdots-\theta_{p} B^{p}
\end{aligned}
$$

Morettin et al. (2006), afirmam que, para uma série que não apresenta características de estacionariedade, se tornar estacionária, é necessário passar por um processo de diferenciação. A partir de um processo de $d$ diferenças, a série se torna estacionária e é nomeada como série integrada de ordem $d$. Após o processo de diferenciação, o modelo $\operatorname{ARMA}(p, q)$, integrado, passa a ser denominado de modelo $\operatorname{ARIMA}(p, d, q)$, que apresenta sua forma na equação 4.

$$
\phi(B)(1-B)^{d} X_{t}=\theta(B) \varepsilon_{t}
$$

Onde $d$ = ordem de integração, número de diferenças necessárias para tornar a série estacionária.

De modo complementar, como exposto por Bayer et al. (2012), em estudos de variáveis ambientais é corriqueiro o encontro de sazonalidades nas séries, ou seja, padrões de repetições nos dados. Deste modo, os modelos ARIMA $(p, d, q)$ são, então, ampliados para os modelos SARIMA $(p, d, q)(P, Q, D)$, definidos na forma geral expressa na equação 5 (MORETTIN et al., 2006).

$$
\phi(B) \Phi(B)(1-B)^{d}\left(1-B^{s}\right) X_{t}=\theta(B) \Theta(B) \varepsilon_{t}
$$

Onde:

$p, d, q=$ ordens do modelo referentes à dinâmica ordinal; $\mathrm{P}, \mathrm{D}, \mathrm{Q}=$ ordens referentes à parte sazonal do modelo.

Os parâmetros $\phi$ e $\vartheta$ são parâmetros autorregressivos e de médias móveis ordinais, e os parâmetros $\Phi$ e $\Theta$ são parâmetros autorregressivos e de medias móveis sazonais (MORETTIN et al., 2006). Para observações mensais, com sazonalidade de 12 meses, tem-se que a periodicidade sazonal (s) é igual a 12.

Para a identificação das ordens de integração d, D e as ordens p, q, P e Q do modelo, utilizou-se a análise dos correlogramas, que são gráficos das funções de autocorrelação (FAC) e de autocorrelação parcial (FACP) amostrais. De acordo com Ehlers (2007), se os correlogramas decaem muito lentamente para zero há, então, a indicação de que a série seja não estacionária. Contudo, um método bastante utilizado para identificar as ordens do modelo se dá na utilização dos critérios de seleção de modelos. A utilização desses critérios faz com que as fases de identificação e estimação do modelo ocorram simultaneamente.

Para a seleção do modelo foi utilizado o comando auto.arima (), presente no pacote Forecast. Esta função retorna o melhor modelo ARIMA, de acordo com os critérios de informação AIC (Akaike Information Criterion) e BIC (Bayesian Information Criterion), ou seja, aquele que apresenta os menores valores para esses critérios. A função auto.arima, segundo exposto por Krispin (2019), automatiza o processo de ajuste do modelo ARIMA identificando se a série é estacionaria e sazonal, definindo os parâmetros para o modelo.

Após a identificação do melhor modelo e da estimativa de seus parâmetros é implementada a análise 
de diagnóstico com objetivo de verificar se o modelo e seus parâmetros representam adequadamente os dados.

Segundo o apresentado por Bayer et al. (2012), o teste de Ljung-Box (LUNG et al., 1978) é utilizado para avaliar a existência de autocorrelação nos erros por meio da autocorrelação residual. Nesse teste estatístico a hipótese nula a ser testada é a de que os erros são um processo ruído branco (white noise), ou seja, não autocorrelacionados. Portanto, ao não rejeitar a hipótese nula, ou seja, quando o p-valor decorrente do teste estatístico é maior do que o nível de significância $\alpha$, o modelo está adequadamente ajustado.

Além dos critérios de seleção de modelos (AIC) e (BIC), comentados anteriormente, outras medidas de qualidade de ajuste foram utilizadas para auxiliar na seleção do modelo que melhor se ajusta à série temporal em estudo e, principalmente, na avaliação da qualidade do ajuste.

As medidas utilizadas neste trabalho foram o erro quadrático médio (EQM) e o coeficiente de Nash Sutcliffe (CNS), apresentadas na tabela 1 , em que $z_{i}$ e $\hat{z}_{i}$ são os valores observados e previstos no instante $i$, respectivamente, e $\bar{Z}$ é a média aritmética da série observada. Em trabalhos de modelagem hidrológica, cujo objetivo seja modelar vazões, o CNS é a métrica mais utilizada para avaliar o desempenho de modelos. É válido citar que, em relação ao EQM, quanto menor os valores apresentados, melhor é o ajuste e; quanto ao CNS, quanto mais próximo de 1 for o valor, melhor foi o ajuste.

Tabela 1: Medidas de qualidade das previsões.

\begin{tabular}{lc}
\hline Critério & Notação \\
\hline Erro quadrático médio - EQM & $\frac{1}{h} \sum_{i=1}^{h}\left(z_{i}-\hat{z}_{i}\right)^{2}$ \\
Coeficiente de Nash Sutcliffe - CNS & $1-\frac{\sum_{i=1}^{h}\left(Z_{i}-\hat{Z}_{i}\right)^{2}}{\sum_{i=1}^{h}\left(Z_{i}-\bar{Z}_{i}\right)^{2}}$ \\
\hline
\end{tabular}

\section{RESULTADOS E DISCUSSÃO}

Com o intuito de facilitar o entendimento da variável vazão, a Tabela 2 apresenta uma análise descritiva das vazões médias mensais observadas na estação pluviométrica Ponte Vicente Goulart no período de janeiro de 2003 a dezembro de 2014.

Tabela 2: Medidas descritivas da variável vazão em estudo.

\begin{tabular}{ll} 
Medida descritiva & Valor \\
\hline Vazão Mínima $\left(\mathrm{m}^{3} / \mathrm{s}\right)$ & 12,96 \\
Vazão Máxima $\left(\mathrm{m}^{3} / \mathrm{s}\right)$ & 733,12 \\
Média $\left(\mathrm{m}^{3} / \mathrm{s}\right)$ & 129,40 \\
Mediana $\left(\mathrm{m}^{3} / \mathrm{s}\right)$ & 83,34 \\
Desvio Padrão $\left(\mathrm{m}^{3} / \mathrm{s}\right)$ & 120,68 \\
Assimetria & 2,07 \\
Coeficiente de Variação (\%) & 93,24 \\
\hline
\end{tabular}

A média das vazões médias mensais foi de $129,40 \mathrm{~m}^{3} / \mathrm{s}$, com elevado desvio padrão de $120,69 \mathrm{~m}^{3} / \mathrm{s}$ e coeficiente de variação correspondente a 93,27\%. Tais valores indicam presença de sazonalidade na série, sendo a média pouco representativa para a série em pauta, como se observa na Figura 6. A vazão média mensal máxima foi registrada no mês de janeiro de 2007 , com valor de $733,12 \mathrm{~m}^{3} / \mathrm{s}$ enquanto a mínima foi 
registrada em outubro de 2014 , com valor de $12,96 \mathrm{~m}^{3} / \mathrm{s}$.

Na Figura 6 é possível observar o gráfico de linha com a série temporal mensal de vazões médias. A série de vazões possui um padrão de variabilidade intra-anual, com períodos de cheias seguidos por períodos de estiagem, caracterizando a presença de sazonalidade. Verifica-se que a série de vazões possui um período de cheias de dezembro a abril e de estiagem de maio a novembro (Figura 7).

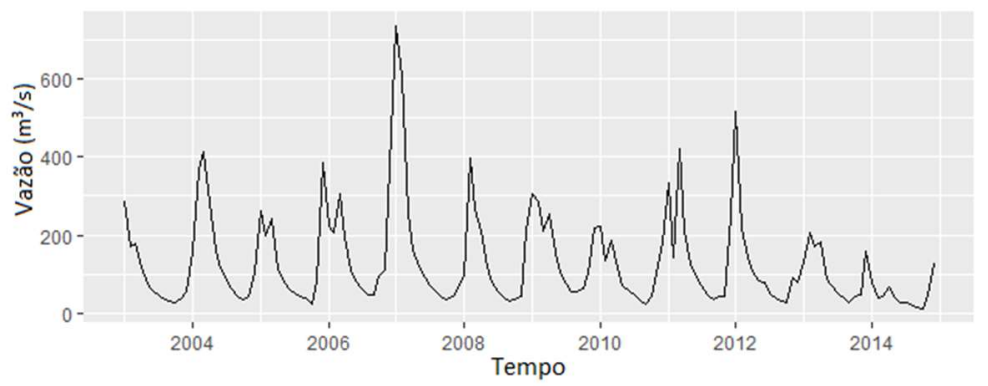

Figura 6: Série de vazões mensais de janeiro de 2003 a dezembro de 2014.

A Figura 7 apresenta o comportamento sazonal da série de vazões médias mensais, agora, de forma a observar o comportamento ao longo dos anos. É notável que o ano de 2014 foi um ano atípico em que as vazões médias apresentaram valores inferiores em comparação aos outros anos da série observada.

A Figura 8 apresenta o gráfico da série vazões médias mensais e sua decomposição em componentes de tendência, sazonalidade e parte aleatória. Nota-se que a série oscila em torno de um valor médio e com um padrão periódico bem definido, o que caracteriza a presença de sazonalidade e a inexistência de tendência nos dados.

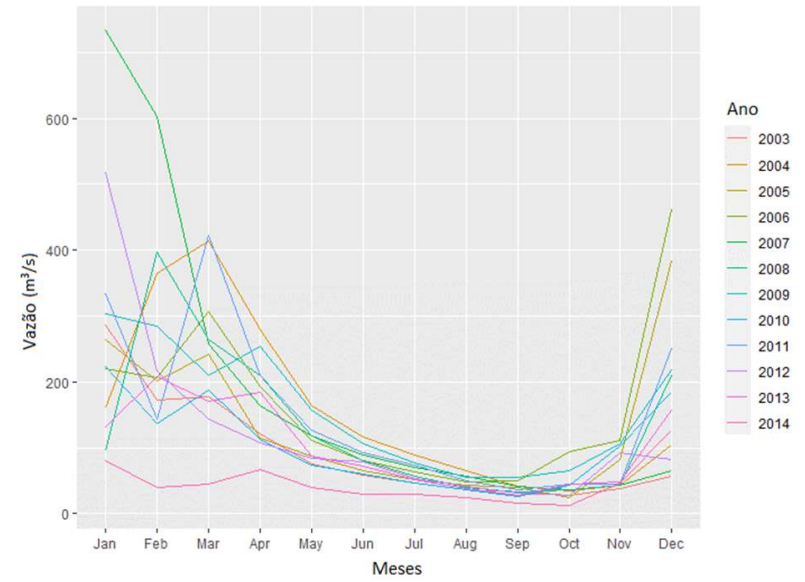

Figura 7: Comportamento sazonal anual das vazões médias mensais.

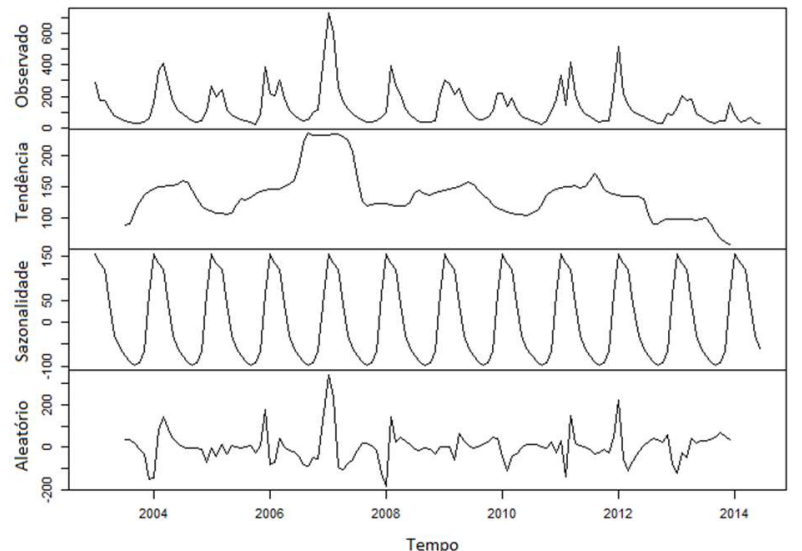

Figura 8: Decomposição da série temporal em componente de sazonalidade, de tendência e de aleatoriedade.

\section{Identificação do Modelo}

A seguir são apresentadas as funções de autocorrelação amostral (FAC) e de autocorrelação parcial amostral (FACP) da série em nível, na Figura 9, e da série diferenciada de ordem 12, na Figura 10.

Analisando-se os correlogramas apresentados na Figura 9a pode-se perceber a autocorrelação significativamente diferente de zero em várias defasagens, principalmente, nas defasagens múltiplas de 12 , indicando sazonalidade da série. Essa componente periódica de 12 meses se reflete no comportamento 
senoidal do referido correlograma, com alta correlação nas lags acima do intervalo de confiança. Do mesmo modo, para a FACP, visto algumas lags apresentarem alta correlação, sugere-se um modelo de médias móveis.
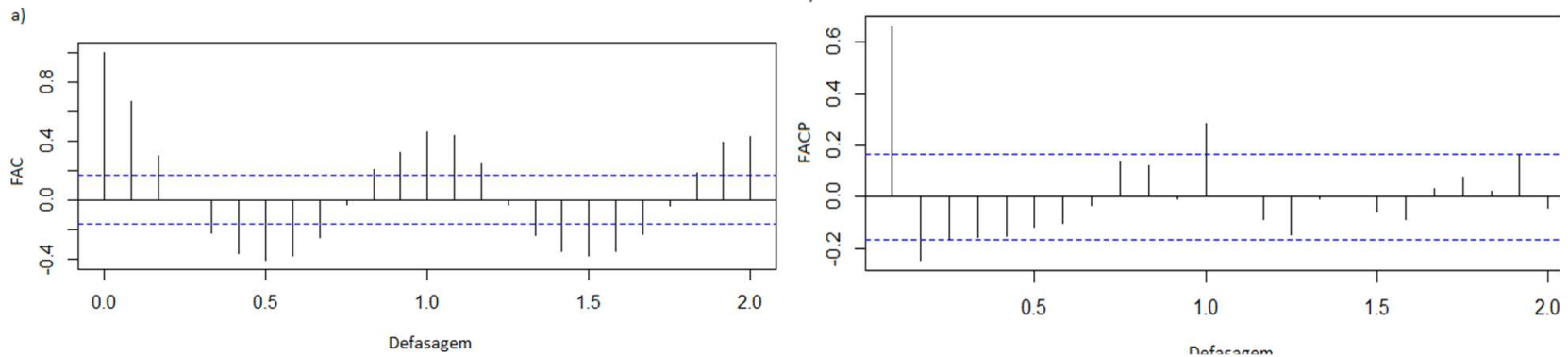

Figura 9: Funções de autocorrelação amostral (FAC) e de autocorrelação parcial amostral (FACP) da série em nível.

Na figura 10, verifica-se as características de modelo estacionário autorregressivo de ordem próxima a 3, pela análise da FAC, e consoante a FACP, de médias móveis também de ordem próxima a 3.
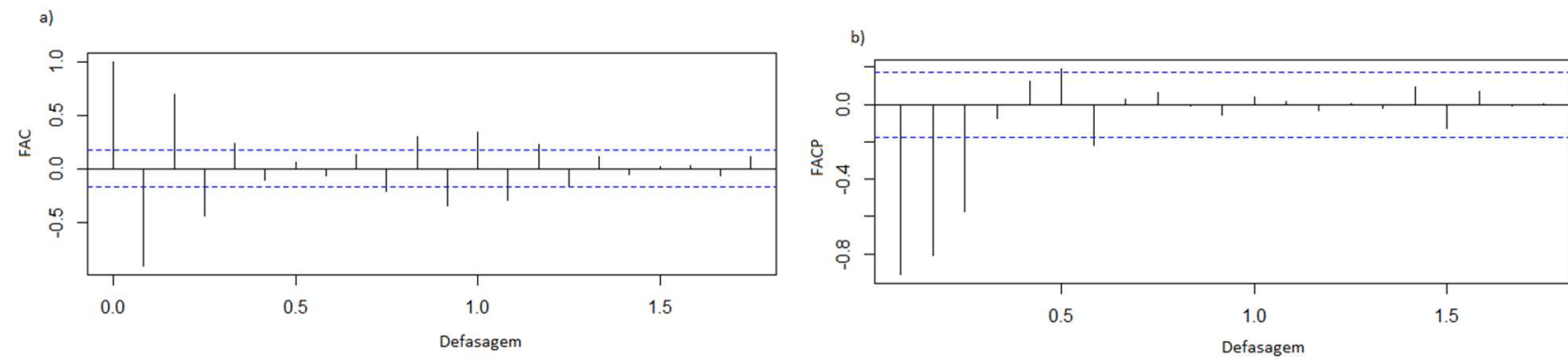

Figura 10: Funções de autocorrelação amostral (FAC) e de autocorrelação parcial amostral (FACP) da série diferenciada de ordem 12.

Portanto, seguindo a referida análise dos correlogramas, o modelo do tipo SARIMA deve ser escolhido, pois considera uma diferença sazonal.

Na etapa de identificação do modelo determinam-se os valores de $p, d, q$ e $P, D$ e $Q$ dos modelos SARIMA e as estimativas dos parâmetros desses modelos. Inicialmente testaram-se todos os possíveis modelos tais que: a ordem autorregressiva ( $p$ e $P$ ) e de médias móveis ( $q$ e $Q$ ), fossem inferiores a 3, conforme análise prévia, e as diferenças ( $d$ e $D$ ) menores que 2.

Para a seleção do melhor modelo foram utilizados a função auto.arima e a análise dos critérios AIC e BIC. A função retornou o modelo SARIMA $(2,1,2)(1,0,0)_{12}$ como o que melhor se ajustou a série apresentada. Para os critérios foram selecionados 10 modelos com os menores valores dos referidos critérios. A Tabela 3 apresenta os modelos estimados para o estudo de previsão da série de vazão média mensal, com os seus respectivos valores de AIC e BIC, em ordem crescente. De acordo com os critérios de AIC e BIC, o modelo SARIMA $(2,1,2)(1,0,0)_{12}$ foi o que melhor se ajustou aos dados em estudo.

Tabela 3: Modelos concorrentes e seus valores de AIC e BIC.

\begin{tabular}{lll}
\hline Modelos SARIMA & & \\
\hline $\mathbf{( p , ~} \mathbf{d}, \mathbf{q})(\mathbf{P}, \mathbf{D}$ e Q) & AIC & BIC \\
$\mathbf{( 2 , 1 , 2 ) ( 1 , 0 , 0 ) [ 1 2 ]}$ & $\mathbf{1 6 1 5 , 8 5 5}$ & $\mathbf{1 6 3 3 , 3 7 5}$ \\
$(2,1,2)(0,0,1)[12]$ & 1616,455 & 1633,975 \\
$(0,1,2)(2,0,0)[12]$ & 1624,838 & 1639,437 \\
$(0,1,2)(0,0,2)[12]$ & 1625,740 & 1640,340 \\
\hline
\end{tabular}




\begin{tabular}{lrr}
$(0,1,2)(3,0,0)[12]$ & 1626,741 & 1644,261 \\
$(0,1,2)(1,0,2)[12]$ & 1627,644 & 1645,163 \\
$(0,1,2)(0,0,3)[12]$ & 1627,648 & 1645,168 \\
$(0,1,2)(0,0,1)[12]$ & 1633,955 & 1645,635 \\
$(2,1,0)(0,0,2)[12]$ & 1640,906 & 1655,506 \\
$(3,1,0)(2,0,0)[12]$ & 1640,831 & 1658,351 \\
\hline
\end{tabular}

\section{Diagnóstico do Modelo}

Com o objetivo de validar o modelo, para traçar previsões, foram realizadas as análises de diagnóstico. A normalidade foi verificada com o uso dos testes de Shapiro-Wilk (SHAPIRO et al., 1965) e a para verificação se os resíduos não são autocorrelacionados foi utilizado o teste de Ljung-Box (LUNG et al., 1978), descritos na Tabela 4

Tabela 4: Testes estatísticos de normalidade e correlação do modelo escolhido.

\begin{tabular}{ll}
\hline Modelo SARIMA $(\mathbf{2}, \mathbf{1}, \mathbf{2})(\mathbf{1}, \mathbf{0}, \mathbf{0})_{\mathbf{1 2}}$ & \\
\hline Teste & $\mathbf{p}$-Valor \\
Shapiro-Wilk & $9,083 \mathrm{e}-12$ \\
Ljung-Box & 0,08881 \\
\hline
\end{tabular}

A Figura 11 apresenta, gráfico da série temporal (a), o correlograma (b) e o histograma (c) do resíduo apresentado para o modelo. Analisando os gráficos, é possível observar que os resíduos são normalmente distribuídos. O correlograma apresenta defasagens com valores próximos de zero, onde seu maior valor é, aproximadamente 0,2 , que indica o bom ajuste do modelo e que os resíduos são não autocorrelacionados. Portanto, a análise residual valida o modelo para o passo de previsões. Desta forma, o modelo descreve adequadamente os dados, validando o seu uso para traçar previsões.

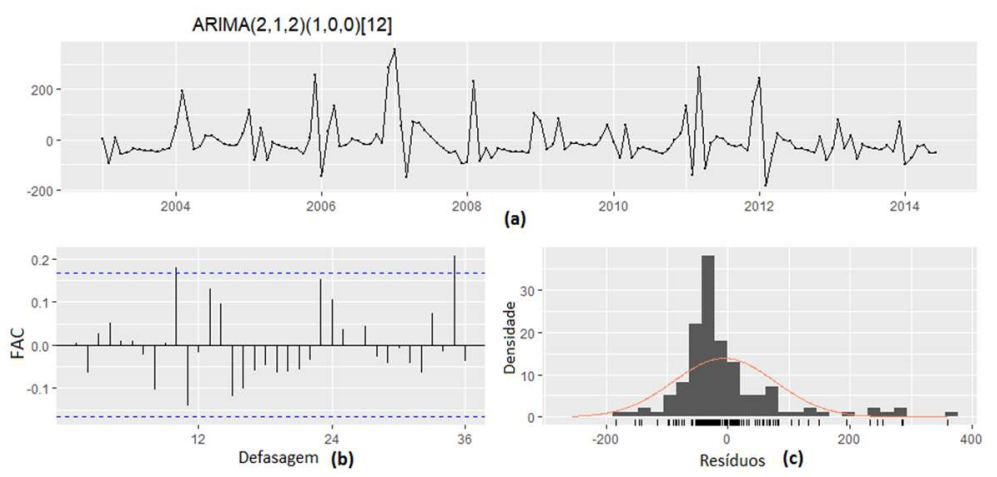

Figura 11: Gráfico da série temporal, correlograma e histograma do resíduo para o modelo.

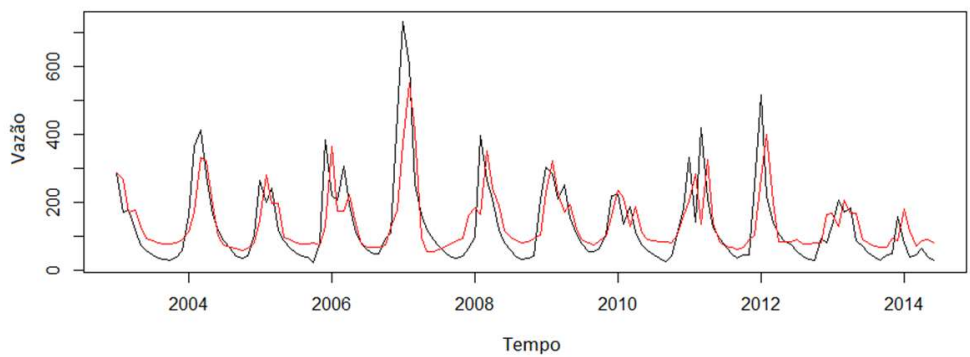

Figura 12: Valores observados (linha preta) e simulados (linha vermelha) da vazão média mensal modelo SARIMA $(2,1,2)(1,0,0) 12$.

Já a Figura 12, apresenta-se a série temporal de vazões médias mensais observadas versus o ajuste dos dados pelo modelo. A partir da análise visual é possível evidenciar o bom desempenho do modelo, 
reforçando os resultados da análise residual confirmando a utilidade do modelo para previsões de vazões. Porém, é possível observar que o modelo superestima as vazões nos períodos de estiagem.

\section{Previsão de vazões}

Na Tabela 5 apresentam-se os valores para o EQM e o NSC para as vazões médias mensais no período de treino e de teste. Como se verifica, o valor para o NSC na etapa de treino apresentou valor de 0,85 , considerado muito bom (MORIASI et al., 2007). Porém, para o teste o valor ficou distante da unidade (aproximadamente, $-3,34$ ), indicando baixo desempenho do modelo para a previsão.

Tabela 5: Previsão e medidas de qualidade de vazões.

\begin{tabular}{lllll}
\hline Mês & Valores observados - Treino & Valores previstos - Treino & Valores observados - Teste & Valores previstos - Teste \\
\hline 1 & 279,57 & 230,22 & - & - \\
2 & 247,75 & 256,89 & - & - \\
3 & 236,69 & 217,65 & - & - \\
4 & 168,14 & 190,40 & - & - \\
5 & 103,52 & 131,93 & - & - \\
6 & 77,44 & 90,55 & - & - \\
7 & 61,69 & 81,26 & 29,59 & 70,987 \\
8 & 46,36 & 76,06 & 25,30 & 103,71 \\
9 & 37,91 & 74,23 & 15,26 & 122,36 \\
10 & 43,87 & 77,43 & 12,96 & 131,62 \\
11 & 68,69 & 91,55 & 46,20 & 128,31 \\
12 & 197,77 & 129,72 & 127,16 & 135,91 \\
NSC & $\mathbf{0 , 8 5}$ & & $-\mathbf{3 , 3 4}$ & \\
EQM & $\mathbf{1 1 0 1 , 7 1}$ & & $\mathbf{3 3 5 2 , 8 8}$ & \\
\hline
\end{tabular}

Os valores observados no teste ilustram que, no ano de 2014, a região passou por uma seca onde as vazões médias mensais foram aquém dos registros anteriores presentes na série histórica. Tal fato relativo ao déficit hídrico juntamente com a vazão máxima registrada para o ano de 2007 podem ser confirmadas pelos registros pluviométricos da estação Vantage Pro 2, localizada na Cooperativa Regional de Cafeicultores em Guaxupé (Cooxupé), município de Rio Paranaíba-MG (SISMET COOXUPÉ, 2020).

Como se observa na Figura 13, os totais precipitados na transição de 2013 para 2014 (222,8; 65,8; $13,6 \mathrm{~mm}$ ) foram inferiores em comparação aos anos anteriores, como se verifica na transição 2012/2013 (241,6; 397,6; 144 mm) e de 2011/2012 (305,4; 392,6; 104,6 mm). Já em relação a máxima vazão observada no ano de 2007 , observou-se para o período a lâmina precipitada de $604,5 \mathrm{~mm}$, confirmando os dados de vazões observados na estação.

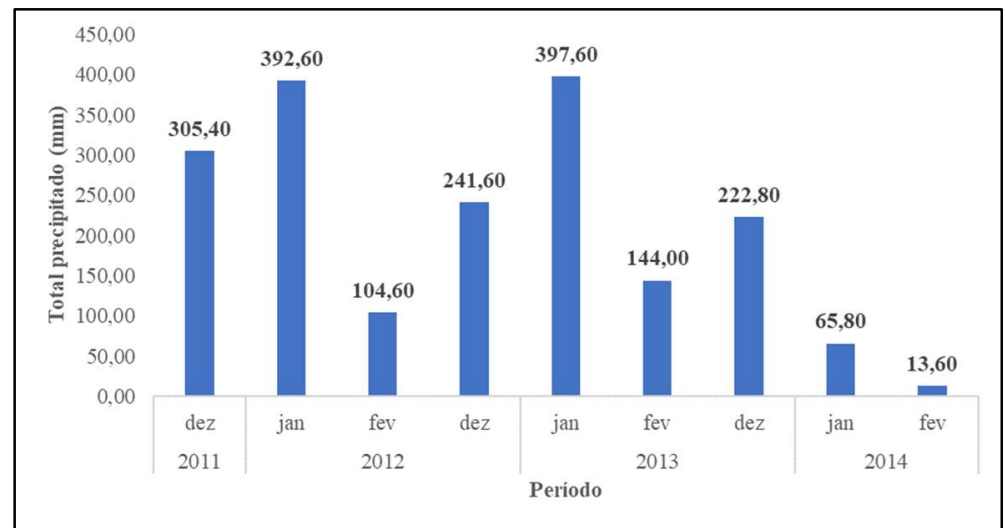

Figura 13: Totais precipitados mensais da estação pluviométrica Cooxupé. 
Como os períodos de estiagem não pertencem a um domínio temporal previamente estabelecido, conforme apontam Reisen et al. (2008), a previsão, realmente, estaria em divergência dos valores observados. Consoante Bleidorn et al. (2019), a ocorrência de um período extremo não pode ser prevista em razão das leis probabilísticas utilizadas em um modelo de previsão.

A estiagem atípica que aconteceu em 2014, levou a uma das nascentes do Rio Paranaíba a secar, e as demais, tiveram uma redução de pelo menos um terço da vazão, conforme relatado pelo presidente do Comitê de Bacia (CBH AMAP); o que reafirma o caráter de excepcionalidade de vazões reduzidas não registradas anteriormente. O modelo utilizado por Bleidorn et al. (2019), executado no rio Jucu, também correlaciona a falha que o modelo apresenta. No referido artigo, o modelo não atingiu resultados satisfatórios, pois na região ocorreram períodos de estiagem, semelhante ao encontrado neste trabalho.

\section{CONCLUSÕES}

No presente trabalho foram realizados ajustes de modelos sazonais da classe ARIMA para uma série de dados de vazões mensais na bacia do rio Paranaíba, na região do Alto Paranaíba. Toda a análise da série temporal em estudo foi realizada usando pacotes do repositório $\mathrm{R}$.

Conclui-se que o modelo que mais se ajustou à série de vazões observadas foi o SARIMA $(2,1,2)(1,0,0)_{12}$. Para seleção do modelo foram utilizados os critérios de seleção AIC e BIC, em que o modelo escolhido apresentou os menores valores para os critérios de 1615,855 e 1633,375, respectivamente. Ao analisar graficamente os valores previstos pelo modelo e os observados na série, verificou-se à similaridade entre os mesmos evidenciando o bom desempenho do modelo.

Porém, para a previsão das vazões no horizonte de seis meses (junho a dezembro de 2014), os valores observados e previstos apresentaram grande discrepância, fato comprovado também pelas medidas de qualidade que apresentaram valores de aproximadamente $-3,34$ para CNS e 3352,88 para EQM. O referido período de baixas vazões fora reforçado pela redução dos totais precipitados na região, por meio da obtenção e análise dos dados pluviométricos. Tais resultados, apontam a limitação de modelos estocásticos na previsão de cenários extremos como a crise hídrica ocorrida; já que se pautam em observações de eventos ocorridos.

Deste modo, recomenda-se para trabalhos futuros a inserção de outras variáveis regressoras em modelos do tipo SARIMAX, no intuito de se avaliar a melhoria dos mesmos em cenários que contemplem eventos extremos.

\section{REFERÊNCIAS}

ANA. Agência Nacional de Águas. Conjuntura dos recursos hídricos no Brasil 2019: informe anual. Brasília: ANA, 2019.

BARBOSA, A. M.; FILL, H. D.. Modelo de previsão de vazão baseado no hidrograma unitário. Revista Brasileira de Recursos Hídricos, Porto Alegre, v.6, n.4, p.165-174, 2001.

BAYER, D. M; CASTRO, M. R; BAYER, F. M.. Modelagem e previsão de vazões médias mensais do rio Potiribu utilizando modelos de séries temporais. Revista Brasileira de Recursos Hídricos. v.17, n.2, p.229-239, 2012.
BLEIDORN, M. T.; PINTO, W. P.; BRAUN, E. S.; LIMA, G. B.; MONTEBELLER, C. A.. Modelagem e previsão de vazões médias mensais do rio Jucu, ES, utilizando o modelo SARIMA. Irriga, Botucatu, v.24, n.2, p.320-355, 2019. DOI: http://doi.org/10.15809/irriga.2019v24n2p320-335

BOX, G. E. P.; JENKINS, G. M.; REINSEL, G. C.. Time series analysis: forecasting and control. 4 ed. Nova Jersey: John Wiely \& Sons, 2008. 
CHECHI, L.; BAYER, F. M.. Modelos univariados de séries temporais para a previsão das temperaturas médias mensais de Erechim, RS. Revista Brasileira de Engenharia Agrícola e Ambiental-Agriambi, v.16, n.12, 2012.

COLLISCHONN, W.; TUCCI, C. E. M.; CLARKE, R. T.; DIAS, P. L. S.; OLIVEIRA, G. S.. Previsão Sazonal de Vazão na Bacia do Rio Uruguai 2: Previsão Climática- Hidrológica. Revista Brasileira de Recursos Hídricos, v.10, n.4, p.61-72, 2005.

CBH AMAP. Comitê da Bacia Hidrográfica dos Afluentes Mineiros do Alto Paranaíba. Plano de ação de recursos hídricos da unidade de gestão hídrica afluentes mineiros do Alto Paranaíba. 2013.

EHLERS, R. S.. Análise de Séries Temporais. 4 ed. Laboratório de Estatística e Geoinformação. Universidade Federal do Paraná, 2007.

IGAM. Instituto Mineiro de Gestão das Águas. Boletim Informativo Bacia do Rio Paranaíba. Belo Horizonte: IGAM, 2015.

KHAIRUDDIN, N.; ARIS, A. Z.; ELSHAFIE, A.; NARANY, T. S.; ISHAK, M. Y.; ISA, N. M.. Efficient forecasting model technique for river stream flow in tropical environment. Urban Water Journal, Kuala Lumpur, v.16, n.8, p.183-192, 2019. DOI: http://doi.org/10.1080/1573062X.2019.1637906

KRISPIN, R.. Hands-on time series analysis with $\mathbf{R}$.

Birmingharm: Packt Publishing, 2019.

LIZZI, E. A. S.. Predição do número mensal de casos de dengue por modelos de séries temporais. Dissertação (Mestrado) - Faculdade de Medicina de Ribeirão Preto, Universidade de São Paulo, Ribeirão Preto, 2012.

LJUNG, G. M.; BOX, G. E. P.. On a measure of lack of fit in time series models. Biometrika, Grã-Bretanha, v.65, n.2, p.297-303, 1978.

MARTÍNEZ, L.; BARBOZA, J. M.; RAMOS, Á. L.; LÓPEZ, J.; LAMBRAÑO, A. L.. SARIMA Approach to Generating Synthetic Monthly Rainfall in the Sinú River Watershed in Colombia. Atmosphere, Bogotá, v.11, n.602, 2020. DOI: http://doi.org/10.3390/atmos11060602
MORETTIN, P. A.; TOLOI, C. M. C.. Análise de series temporais. 2 ed. São Paulo: Egard Blucher, 2006.

MORIASI, D. N.; ARNOLD, J. G.; LIEW, M. W. V.; BINGER, R. L.; HARMEL, R. D.; VEITH, T.. Model evaluation guidelines for systematic quantification of accuracy in watershed simulations. Transactions of the ASABE, v.50, p.885-900, 2007.

UNESCO. Organização das Nações Unidas para a Educação, a Ciência e a Cultura. Relatório mundial das nações unidas sobre o desenvolvimento dos recursos hídricos 2019. Brasil: UNESCO, 2019.

PINTO, W. P.; LIMA, G. B.; ZANETTI, J. B.. Análise comparativa de modelos de séries temporais para modelagem e previsão de regimes de vazões médias mensais do Rio Doce, Colatina - Espírito Santo. Ciência e Natura, Santa Maria, v.37, n.3, p.1-11, 2015. DOI: http://doi.org/10.5902/2179460X17143

REISEN, V. A.; MOLINARES, F. A.; TEIXEIRA, E. C.. Modelagem de séries temporais sazonais na presença de outliers: estudo de caso da vazão máxima mensal do rio Jucu, ES, Brasil. Revista Brasileira de Recursos Hídricos, Porto Alegre, v.13, n.2, p.45-53, 2008

SANTOS, P. G. P.; OLIVEIRA, T. F.. Modelagem e previsão de vazão afluente média mensal no Rio Tocantins, Usina Hidrelétrica Tucuruí-Pará, Amazônia, Brasil. Biota Amazônia, Macapá, v.6, n.2, p.9-16, 2016. DOI: http://doi.org/10.18561/2179-5746/biotaamazonia.v6n2p9$\underline{16}$

SHAPIRO, S. S.; WILK, M. B.. An analisy of variance test for normality (complete samples). Biometrika, Grã-Bretanha, v.52, p.591-611, 1965.

SISMET COOXUPÉ. Dados dos pontos pluviométricos. Rio Paranaíba, 2020.

TUCCI, C. E. M.. Modelos hidrológicos. Porto Alegre: UFRGS 1998.

WERNER, L.; BISOGNIN, C.; MENEGOTTO, L.. Previsão da umidade relativa do ar diária de Porto Alegre. Cadernos do IME - Série Estatística, v.43, p.39-59, 2017, DOI: http://doi.org/10.12957/cadest.2017.31211

A CBPC - Companhia Brasileira de Produção Científica (CNPJ: 11.221.422/0001-03) detém os direitos materiais desta publicação. Os direitos referem-se à publicação do trabalho em qualquer parte do mundo, incluindo os direitos às renovações, expansões e disseminações da contribuição, bem como outros direitos subsidiários. Todos os trabalhos publicados eletronicamente poderão posteriormente ser publicados em coletâneas impressas sob coordenação da Sustenere Publishing, da Companhia Brasileira de Produção Científica e seus parceiros autorizados. Os (as) autores (as) preservam os direitos autorais, mas não têm permissão para a publicação da contribuição em outro meio, impresso ou digital, em português ou em tradução. 